Vol. 3, No. 3, July 2018, 119-122

Webpage: http://rheumres.org

\title{
Human leukocyte antigen-DRB1 alleles in patients with sarcoidosis from Northeast Iran
}

\author{
SeyedehZahra Mirfeizi ${ }^{1}$, Maryam Sahebari ${ }^{1}$, Shima Nabavi ${ }^{1}$, Masoumeh Salari ${ }^{1}$, Masoud Saghafi ${ }^{1}$, Zahra \\ Rezaieyazdi ${ }^{1}$, Narges Valizadeh ${ }^{2}$, Houshang Rafatpanah ${ }^{2 *}$ \\ ${ }^{1}$ Rheumatic Diseases Research Center, Faculty of Medicine, Mashhad University of Medical Sciences, Mashhad, Iran; ${ }^{2}$ Immunology \\ Research Center, Inflammation and Inflammatory Diseases Division, Mashhad University of Medical Sciences, Mashhad, Iran
}

\begin{abstract}
Sarcoidosis a systemic granulomatous disorder of unknown etiology with varying clinical pictures. HLA genes, especially HLADRB1, have been shown to be candidates for the etiology of sarcoidosis. This study examined the association between the polymorphism of HLA-DRB1 alleles and sarcoidosis in Iranian subjects. The study population included 58 patients with sarcoidosis and 68 healthy controls. Genomic DNA was extracted, and the polymorphisms of the HLA-DRB1 alleles were determined using a polymerase chain reaction with sequence-specific primer (PCR-SSP). The frequency of HLA-DRB1 *07 was higher in sarcoidosis patients $(25.8 \%)$ than in controls (15.3\%); however no significant difference was observed between the two groups $(P>0.05)$. The frequency of HLA-DRB $1 * 11$ was higher in the control group $(31.9 \%)$ than in cases $(22.4 \%)$, but no significant difference was detected between the groups $(P>0.05)$. The results of the present study showed that there is no association between HLA-DRB1 alleles and susceptibility to sarcoidosis in Mashhad, Northeast Iran. Further studies with large sample sizes are required in order to clarify this issue.
\end{abstract}

Keywords: HLA-association study, HLA DRB1, PCR-SSP, sarcoidosis

\section{Introduction}

Sarcoidosis is a systemic granulomatous disorder of unknown etiology origin, characterized by an accumulation of effector $\mathrm{T}$ lymphocytes and the presence of non-caseating epithelioid granulomas in affected organs $[1,2]$. Accumulated data has suggested that aberrant immune responses to environmental and infectious factors are involved in the pathophysiology of disease in hosts that are genetically susceptible [3].

There are several lines of evidence that strongly support the genetic susceptibility to sarcoidosis, such as the high concordance rates for the disease in monozygotic twins when compared to dizygotic, familial and ethnic clustering of the disease and significant differences in the prevalence and clinical manifestation of the disease in different geographic areas and ethnic groups [4-6].

Linkage and association studies across different ethnic populations have suggested that alleles of human leukocyte antigen (HLA) class I and class II alleles are associated with increased risks of sarcoidosis [7, 8]. Although HLA class II alleles are the major contributors to disease susceptibility, a large number of genes are thought to contribute to causing disease $[9,10]$.

Amongst the HLA class II gene, the variation in the HLA-DRB1 gene has the most impact and influence in both the susceptibility and prognosis of sarcoidosis [11]. It has been shown that HLA-DRB $1 * 01$ and DRB $1 * 04$ alleles exhibit protective effects for sarcoidosis, while other alleles such as DRB1*03, DRB1*11, DRB1*12, $\mathrm{DRB} 1 * 14$, and DRB $1 * 15$ are associated with an increased risk for disease [12].

In Japanese patients, Ishihara et al. reported that HLADRB1*11, -DRB1*14, and -DRB1*08 alleles increased the risk of sarcoidosis [13]. The HLADRB $1 * 150101$ allele has been shown to be a positive marker for severe pulmonary sarcoidosis in Dutch Caucasian patients [14]. In a study of Swedish patients, HLA-DRB $1 * 03$, HLADRB $1 *$ 14 , and HLA-DRB $1 * 15$ were shown to be associated with the disease, while HLA-DRB $1 * 01$ and HLA-DRB $1 * 04$ were identified as protective factors against sarcoidosis [15]. In a Chinese Han population, Ying et al. reported that the HLA-DRB $1 * 11$ allele is associated with sarcoidosis, whereas the HLA-DRB $1 * 07$ allele decreased the risk of

\footnotetext{
* Corresponding Author: Houshang Rafatpanah, E-mail: rafatpanahh@mums.ac.ir, Tel: +98-51-38002379
}

Received: 29 January 2018; Accepted: 1 May 2018 
HLA and sarcoidosis

disease [11]. Furthermore, it has been suggested that HLADQB1 but not HLA-DRB1 plays a pivotal role in sarcoidosis susceptibility in African Americans [16].

Data accumulated from different populations has produced conflicting results regarding the role of HLA in sarcoidosis; however, it would be informative to examine the impact of HLA on people of different ethnic backgrounds. In the present study, the frequency of HLADRB*1 alleles in a Caucasian population of Mashhad in northeast Iran was investigated.

\section{Materials and Methods}

\section{Study population}

The study population included 58 patients who referred to Ghaem and Imam Reza Hospitals, Mashhad University of Medical Sciences, Mashhad, Iran, between January 2011 and August 2013. All patients were examined using the clinical diagnostic criteria for sarcoidosis according to the American Thoracic Society consensus panel [2]. The inclusion criteria were established based on the presence of clinical symptoms, radiological fractures, and pathological data, while patients with a differential diagnosis such as tuberculosis (TB) or fungal infection were excluded from the study.

The mean age of patients was $43 \pm 8$ years (range: 30 to 62 years), of which $76.2 \%$ were women and $27.6 \%$ were men. Sixty-eight of the age- and gender-matched controls were selected $(P=0.1)$ from the Mashhad Blood Transfusion Center as previously described [17]. The study was approved by the Ethics Committee of Mashhad University of Medical Sciences, Mashhad, Iran, and a signed consent form was obtained from all subjects.

\section{HLA-DRB1 typing}

Four milliliters of blood were taken from all cases and DNA was extracted using a standard phenol-chloroform method. The frequencies of HLA-DRB1 alleles were determined in all subjects by applying a polymerase chain reaction with PCR-SSP using a commercial method (BAGS, Germany) according to the manufacturers' instruction.

\section{Statistical analysis}

Differences in HLA-DRB1 allele frequencies between patients and the control group were analyzed using STATA version 10 (STATA Corp.). The level of statistical differences in HLA-DRB1 allele frequencies between the two studied groups was applied by the Fisher's exact test. Odds ratios (OR) and $95 \%$ confidence intervals (CI) were used to evaluate the strength of association of alleles. A pvalue of less than 0.05 was considered to be significant.

\section{Results}

The population studied consisted of 58 patients with sarcoidosis and 68 healthy controls. A history of sarcoidosis was observed in only two patients (3.4\%). The symptoms observed in patients were arthritis (96.6\%), erythema nodosum $(63.8 \%)$, respiratory symptoms $(24.1 \%)$, skin lesions (5.2\%), uveitis (15.5\%), fatigue (48.3\%), fever $(27.6 \%)$, night sweats $(3.4 \%)$, and weight loss $(1.7 \%)$. Only one patient had sarcoidosis with vitiligo. In $93.4 \%$ of patients, lymphadenopathy was observed by chest X-ray, while by CT scanning lymphadenopathy was diagnosed in $92.5 \%$ of the patients (Table 1). The mean level of angiotensin-converting enzyme (ACE) in patients was $53.6 \pm 32.1$.

Table 1. Clinical characteristics of patients with sarcoidosis

\begin{tabular}{lcc}
\hline Clinical symptoms & N & \% \\
\hline Arthritis & 56 & 96.6 \\
Erythemanodosum & 37 & 63.8 \\
Respiratory symptoms & 14 & 24.1 \\
Skin lesions & 3 & 5.2 \\
Uveitis & 9 & 15.5 \\
Fatigue & 28 & 48.3 \\
Fever & 16 & 27.6 \\
Sweat nights & 2 & 3.4 \\
Weight loss & 1 & 1.7 \\
Lymphadenopathy in lung umbilicus & 54 & 93.4 \\
\hline
\end{tabular}

The HLA-DRB1*07 allele was more frequent in patients with sarcoidosis $(25.8 \%)$ in comparison with control group (15.3\%); however, no significant difference was observed between the two groups $(P>0.05)$. In contrast, the HLA-DRB $1 * 11$ allele was more frequent in the control group $(31.9 \%)$ than in patients $(22.4 \%)$, but no significant difference was detected between both groups $(P>0.05)$. Furthermore, no significant differences in HLADRB1 allele frequencies were found between patients and controls $(P>0.05$; Table 2).

\section{Discussion}

Genetic and environmental factors seem to be associated with the pathogenesis of sarcoidosis. Among the genetic factors, HLA class I and II molecules, which present antigenic proteins to the T-cell receptors, are involved in the development of sarcoidosis [18].

Several studies have demonstrated the association of HLA class II alleles with sarcoidosis is influenced by region, race, and country [19]. 
Table 2. HLA-DRB1 phenotype frequencies in sarcoidosis patients and healthy controls

\begin{tabular}{|c|c|c|c|c|c|c|}
\hline \multirow[t]{2}{*}{ HLA DRB1 allele } & \multicolumn{2}{|c|}{$\begin{array}{c}\text { Patients } \\
\text { N=58 }\end{array}$} & \multicolumn{2}{|c|}{$\begin{array}{c}\text { Controls } \\
\mathrm{N}=68\end{array}$} & \multirow[t]{2}{*}{ OR $(95 \%$ CI $)$} & \multirow[t]{2}{*}{$p$ value } \\
\hline & $\mathbf{n}$ & $\%$ & n & $\%$ & & \\
\hline DRB $1 * 01$ & 5 & 8.6 & 8 & 11.1 & $0.7(0.17-2.63)$ & 0.56 \\
\hline $\mathrm{DRB} 1 * 03$ & 11 & 18.9 & 12 & 16.7 & $1.09(0.39-2.98)$ & 0.84 \\
\hline DRB1*04 & 16 & 27.5 & 19 & 26.4 & $0.98(0.41-2.30)$ & 0.96 \\
\hline $\mathrm{DRB} 1 * 05$ & 0 & - & 0 & - & - & - \\
\hline DRB1*06 & 0 & - & 0 & - & - & - \\
\hline $\mathrm{DRB} 1 * 07$ & 15 & 25.8 & 11 & 15.3 & $1.61(0.61-4.32)$ & 0.18 \\
\hline DRB $1 * 08$ & 2 & 3.5 & 1 & 1.4 & $2.39(0.12-143.2)$ & 0.46 \\
\hline DRB $1 * 09$ & 1 & 1.7 & 4 & 5.6 & $0.28(0.005-2.96)$ & 0.23 \\
\hline DRB $1 * 10$ & 1 & 1.7 & 3 & 4.2 & $0.38(0.007-4.91)$ & 0.69 \\
\hline DRB $1 * 11$ & 13 & 22.4 & 23 & 31.9 & $0.56(0.23-1.33)$ & 0.15 \\
\hline DRB1*12 & 1 & 1.7 & 3 & 4.2 & $0.38(0.007-4.91)$ & 0.69 \\
\hline DRB $1 * 13$ & 23 & 43 & 25 & 34.7 & $1.13(0.051-2.47)$ & 0.73 \\
\hline DRB $1 * 14$ & 4 & 6.8 & 5 & 6.9 & $0.93(0.17-4.58)$ & 0.92 \\
\hline DRB $1 * 15$ & 10 & 29.3 & 13 & 18.1 & $0.88(0.31-2.4)$ & 0.78 \\
\hline DRB1*16 & 1 & 1.9 & 6 & 8.3 & $0.18(0.003-1.58)$ & 0.82 \\
\hline
\end{tabular}

In the present study, the frequency of the HLA$\mathrm{DRB} 1 * 07$ allele was higher in patients with sarcoidosis than those in the control group; however, no significant difference was observed between the two groups. Previous Association studies have shown that HLA-DRB1*03, DRB1*11, DRB1*12, DRB1*14, and DRB1*15 alleles increase the risk of sarcoidosis, while HLA-DRB $1 * 01$ and DRB $1 * 04$ alleles act as protective factors against the disease [10].

Mortaz et al. reported that the frequency of the HLADRB $1 * 07$ allele was significantly higher in control subjects than sarcoidosis patients in the center of Tehran, Iran, and the patients with this allele showed good response to treatment. The frequency of the HLA-DRB $1 * 12$ allele, however, was increased in patients compared with healthy controls. Other studies have also suggested that a good sarcoidosis prognosis is associated with the HLADRB $1 * 7$ allele $[20,21]$.

In contrast, the present study demonstrated that the HLADRB $1 * 7$ allele was more frequent in patients compared with the controls; however, no significant difference was observed between the two groups. Consistent with the current results, Swedish sarcoidosis patients show an increased frequency of HLA-DR *7 [22].

In the current study, the HLA-DRB $1 * 11$ allele was more frequent among control subjects than sarcoidosis patients; however, no significant difference was observed between the groups. Thus, it is likely that this allele is a protective factor for sarcoidosis. Ozyilmaz et al. reported high HLADRB $1 * 11$ allele frequency in patients of a Turkish Caucasian population without extra-pulmonary sarcoidosis, suggesting that this allele could be a protective factor for systemic involvement [23].

In their study, da Costa et al. examined the HLA alleles in Brazilian sarcoidosis patients. The result of their study showed that the DRB1*16 allele was more frequently found in control groups compared to sarcoidosis patients. However, no significant differences were found between sarcoidosis patients and controls, which is consistent with the current results [24]. Ozyilmaz et al. reported a high frequency of the HLA DRB $1 * 15$ allele in sarcoidosis patients than in the control group, and further multivariate analysis suggested the presence of this allele is a risk factor for sarcoidosis [24].

Genetic association studies in sarcoidosis have shown conflicting results, and discrepancies in HLA and HLA$\mathrm{DRB} 1 *$ in the disease have also been reported across different ethnic groups. It is more likely that the relationship between environmental agents and an environment gene plays a pivotal role in complex diseases such as sarcoidosis. The current study has some limitations, such as the relatively small sample size of the studied population, and there is no available data regarding the exposure to the environmental factors, which are more likely needed for a predisposition to sarcoidosis.

In conclusion, no association between HLADRB1alleles and sarcoidosis were found in the present study. Further studies are needed to evaluate the prognostic value of the MHC in larger cohorts in Iranian populations.

\section{Conflicts of interest}

The authors declare no conflict of interest.

\section{Acknowledgments}

This study was supported by a grant from Mashhad University of Medical Sciences, Mashhad, Iran. 
HLA and sarcoidosis

\section{References}

1. Newman LS, Rose CS, Maier LA. Sarcoidosis. N Engl J Med 1997; 336(17): 1224-34. doi: 10.1056/ nejm199704243361706.

2. Statement on sarcoidosis. Joint statement of the American Thoracic Society (ATS), the European Respiratory Society (ERS) and the World association of Sarcoidosis and Other Granulomatous Disorders (WASOG) adopted by the ATS Board of Directors and by the ERS Executive Committee. Am J RespirCrit Care Med 1999; 160(2): 736-55. doi: 10.1164/ajrccm.160.2. ats4-99.

3. Newman LS, Rose CS, Bresnitz EA, Rossman MD, Barnard J, Frederick $\mathrm{M}$, et al. A case control etiologic study of sarcoidosis: A case control etiologic study of sarcoidosis: environmental and occupational risk factors. Am J Respir Crit Care Med 2004; 170(12): 1324-30. doi: 10.1164/rccm.200402-249oc.

4. Sverrild A, Backer V, Kyvik KO, Kaprio J, Milman N, Svendsen CB, et al. Heredity in sarcoidosis: a registry-based twin study. Thorax 2008; 63(10): 894-96. doi: 10.1136/thx.2007.094060.

5. Rybicki BA, Kirkey KL, Major M, Maliarik MJ, Popovich J Jr, Chase GA et al. Familial risk ratio of sarcoidosis in African-American sibs and parents. Am J Epidemiol 2001; 153(2): 188-93. doi: 10.1093/aje/ 153.2.188.

6. Hosoda Y, Yamaguchi M, Hiraga Y. Global epidemiology of sarcoidosis. What story do prevalence and incidence tell us? Clin Chest Med 1997; 18(4): 681-94. doi: 10.1016/s0272-5231(05)70412-3.

7. Grunewald J. Genetics of sarcoidosis. Curr Opin Pulm Med 2008; 14(5): 434-9. doi: 10.1097/ mcp.0b013e3283043de7.

8. Spagnolo P, du Bois RM. Genetics of sarcoidosis. Clin Dermatol 2007; 25(3): 242-9. doi: 10.1016/j. clindermatol.2007.03.001.

9. Grunewald J. Role of genetics in susceptibility and outcome of sarcoidosis. Semin Respir Crit Care Med 2010; 31(04): 380-9. doi: $10.1055 / \mathrm{s}-0030-1262206$.

10. Spagnolo P, Grunewald J. Recent advances in the genetics of sarcoidosis. J Med Genet 2013; 50(5): 290-297. doi: 10.1136/ jmedgenet-2013-101532.

11. Zhou Y, Shen L, Zhang Y, Jiang D, Li H. Human leukocyte antigen-A, $\mathrm{B}$, and -DRB 1 alleles and sarcoidosis in Chinese Han subjects. Hum Immunol 2011; 72(7): 571-5. doi: 10.1016/j.humimm.2011.03.020.

12. Grutters JC, Sato H, Welsh KI, du Bois RM. The importance of sarcoidosis genotype to lung phenotype. Am J Respir Cell MolBiol 2003; 29: S59-62.

13. Ishihara $\mathbf{M}$, Ishida $\mathrm{T}$, Inoko $\mathrm{H}$ et al. HLA serological and class II genotyping in sarcoidosis patients in Japan. Jpn J Ophthalmol 1996; 40(1): 86-94.

14. Voorter CE, Drent M, van den BergLoonen EM. Severe pulmonary sarcoidosis is strongly associated with the haplotype HLADQB1*0602-DRB1*150101. Hum Immunol 2005; 66(7): 826-35. doi: 10.1016/j.humimm.2005.04.003.

15. Grunewald J, Brynedal B, Darlington P, Nisell M, Cederlund K, Hillert J et al. Different HLA-DRB1 allele distributions in distinct clinical subgroups of sarcoidosis patients. Respir Res 2010; 11(1): 25. doi: 10.1186/1465-9921-11-25.

16. Rybicki BA, Maliarik MJ, Poisson LM, Sheffer R, Chen KM, Major M et al. The major histocompatibility complex gene region and sarcoidosis susceptibilityin African Americans. Am J RespirCrit Care Med 2003; 167(3): 444-9. doi: $10.1164 / \mathrm{rccm}$. 2112060.

17. Rafatpanah $\mathrm{H}$, Pravica $\mathrm{V}$, Faridhosseini R, Tabatabaei A, Ollier
$\mathrm{W}$, Poulton $\mathrm{K}$ et al. Association between HLA-DRB $1 * 01$ and HLA$\mathrm{CW}^{*} 08$ and outcome following HTLV-I infection. Iran J Immunol 2007; 2(4): 94-100. doi: IJIv4i2A4.

18. Moller DR, Chen ES. What causes sarcoidosis? Curr Opin Pulm Med 2002; 8(5): 429-34. doi: 10.1097/ 00063198-200209000-00015.

19. Grunewald J. Review: role of genetics in susceptibility and outcome of sarcoidosis. Semin Respir Crit Care Med 2010; 31(4): 380-9. doi: 10.1055/s-00301262206.

20. Berlin M, Fogdell-Hahn A, Olerup O, Eklund A, Grunewald J. HLA-DR predicts the prognosis in Scandinavian patients with pulmonary sarcoidosis. Am J RespirCrit Care Med 1997; 156(5): 1601-5. doi: 10.1164/ajrccm.156.5. 9704069.

21. Planck A, Eklund A, Yamaguchi E, Grunewald J. Angiotensin converting enzyme gene polymorphism in relation to HLADR in sarcoidosis. J Intern Med 2002; 251(3): 217-22. doi: 10.1046/j.1365-2796.2002.00946.x.

22. Papadopoulos KI, Wassmuth R, Sponsel T, Sjöberg K, Hallengren B. Sarcoidosis and autoimmunity: evidence of differential associations with HLA class II markers. Int Endocrine Metab 2006; 4(1): 13-8.

23. Ozyilmaz E, Goruroglu Ozturk O, Yunsel D, Deniz A, Hanta I, Kuleci $S$ et al. Could HLA-DR B1*11 allele be a clue for predicting extrapulmonary sarcoidosis? Sarcoidosis Vasc Diffuse Lung Dis 2014; 31(2): 154-62.

24. da Costa CH, Silva VL, FabricioSilva GM, Usnayo M, Rufino R, Porto LC. HLA in a cohort of Brazilian patients with sarcoidosis. Hum Immunol 2013; 74(10): 132632. doi: 10.1016/j.humimm.2013.07. 009. 